\title{
Analysis of the clinical significance of DCLKI+ colorectal cancer using novel monoclonal antibodies against DCLKI
}

This article was published in the following Dove Press journal:

OncoTargets and Therapy

\author{
Tianqi Dai' \\ Yunlong $\mathrm{Hu}^{\mathrm{l}-3}$ \\ Fulian Lv' \\ Tatsuhiko Ozawa ${ }^{2}$ \\ Xin Sun' \\ Jingjing Huang' \\ Xiaojian Han' \\ Hiroyuki Kishi \\ Atsushi Muraguchi² \\ Aishun Jin' \\ 'Department of Immunology, College \\ of Basic Medical Sciences, Harbin \\ Medical University, Harbin, People's \\ Republic of China; ${ }^{2}$ Department of \\ Immunology, Graduate School of \\ Medicine and Pharmaceutical Sciences, \\ University of Toyama, Toyama, Japan; \\ ${ }^{3}$ Department of Pathogen Biology, \\ Shenzhen University School of \\ Medicine, Shenzhen, People's Republic \\ of China
}

Correspondence: Aishun Jin Department of Immunology, College of Basic Medical Sciences, Harbin Medical University, No 194 Xuefu Road, Harbin, People's Republic of China Tel +86 I53 20375616 Fax +86 45I 86697322

Email aishunjin@ems.hrbmu.edu.cn
Introduction: Doublecortin-like kinase 1 (DCLK1) is considered a putative tumor stem cell (TSC) marker and a promising therapeutic target, as DCLK $1^{+}$progeny cells exhibit high expression in tumors. However, the biological function of DCLK $1^{+}$cells in tumorigenesis and tumor progression remains unclear.

Materials and methods: We generated rabbit monoclonal antibodies (mAbs) against DCLK1, DCLK1-42, and DCLK1-87 mAbs, using a novel chip-based immunospot array assay on a chip system. First, the specificity of two mAbs to DCLK1 was confirmed by Western blot, which were bound to DCLK1-long in normal colon cells and to DCLK1-short in a cancer cell line as well as colorectal cancer (CRC) cells.

Results: Precise localization analysis using immunofluorescence revealed that both $\mathrm{mAbs}$ had cytoplasmic signal and exhibited a high degree of overlap with microtubules. Furthermore, bacterial display technology indicated that the antigenic epitope region of DCLK1-87 mAb was consistent with that of a commercial anti-DCLK1 polyclonal antibody. In addition, DCLK1-42 mAb has the common polyclonal antibody characteristic of binding to more than one site on DCLK1. By immunohistochemistry, it was found that DCLK1-87 mAb was more specific for DCLK1 ${ }^{+}$cell labeling than a commercial anti-DCLK1 polyclonal antibody. DCLK1 labeled with DCLK1-87 $\mathrm{mAb}$ might be a potential TSC marker because the tissue expression site covers the ALDH1 area in CRC tissues. Finally, we analyzed 100 pairs of cancer tissues and matching paracancerous tissue samples from patients with CRC who received 100 months of follow-up with the DCLK1-87 mAb. The results showed that patients with high DCLK1 expression exhibited a longer survival time than that of patients with low DCLK1 expression $(P=0.0029)$.

Discussion: Our results indicated that we successfully generated an efficient tool for the precise detection of DCLK $1^{+}$cells in cancer tissues. Moreover, we found that high DCLK1 expression in CRC patients appears to play a protective role against tumor progression.

Keywords: doublecortin-like kinase 1, DCLK1 monoclonal antibodies, epitope, colorectal cancer, prognosis

\section{Introduction}

Doublecortin-like kinases (DCLKs) were initially studied in the nervous system. ${ }^{1,2}$ DCLK1, which is a member of the protein kinase superfamily and the doublecortin family, is overexpressed in many cancers, including colon, pancreatic, liver, and esophageal cancers. $^{3-6}$ Based on the lineage tracing of DCLK $1^{+}$cells and their progeny in mice, Nakanishi et al recently showed that DCLK1 is a specific marker of tumor stem cells (TSCs) that do not label normal intestinal stem cells. Furthermore, targeting DCLK1 ${ }^{+}$ cells in mouse models results in tumor regression without discernable effects on the 
adjacent normal tissues. ${ }^{7}$ These findings have shown that DCLK1 is a promising marker for monitoring tumor state and for therapeutic targeting. However, recent reports assessing the biological relevance of $\mathrm{DCLK} 1^{+}$cells to tumor progression and the potential of DCLK1 as a stem cell marker have been inconsistent. $^{8-10}$

Several splice variants of DCLK have been reported, ${ }^{11}$ and a number of long ( $~ 80-82 \mathrm{kDa})$ and short $(\sim 45-50 \mathrm{kDa})$ DCLK1 isoforms have been identified in human neurons. The amino acid (aa) sequence of DCLK1-short (DCLK1-S) also showed $>98 \%$ homology with the C-terminus of DCLK1-long (DCLK1-L). ${ }^{12}$ Human colon cancer cells and colorectal cancer (CRC) cells express DCLK1-S (isoform 2 in the National Center for Biotechnology Information [NCBI] database) transcribed from the $\beta$-promoter in intron $\mathrm{V}$ of the gene, but not DCLK1-L (isoform 1 in the NCBI database) transcribed from the $5^{\prime} \alpha$-promoter. As previously reported, DCLK1-S but not DCLK1-L might serve as a specific cancer cell marker in humans. ${ }^{13}$ Based on preliminary speculation using epitope prediction tools from the Immune Epitope Database, some DCLK1 epitopes are within the 640-729-aa region, because the immunogens of commercially generated monoclonal antibodies (mAbs) against DCLK1 are peptides in the 640-729-aa region. However, additional studies examining the biological functions of DCLK1 in tumor progression have employed polyclonal antibodies against DCLK $1 .{ }^{14}$ Thus, the limited availability of DCLK1-specific antibodies restricts the extensive study of DCLK1 as a potential human cancer cell marker and reduces the credibility of correlations between DCLK1 and tumor progression and prognosis.

In this study, we used two specific mAbs against DCLK1, DCLK1-42 mAb and DCLK1-87 mAb, which were generated using our novel technology to biologically characterize DCLK1 expression in CRC cells and tissues. ${ }^{15}$ We also investigated whether these antibodies were reliable tools for precisely detecting DCLK $1^{+}$cells in cancer tissue, which should be valuable in disease diagnosis and prognosis evaluation.

\section{Materials and methods}

\section{Clinical specimens}

Human tissues were obtained from CRC patients at the First Affiliated Hospital of Harbin Medical University, Harbin, People's Republic of China. This research was approved by the Ethics Committee of Harbin Medical University. Written informed consent was obtained from all patients. This tissue chip study was approved by the National Human Resources Sharing Service Platform, number 2005DKA21300.

\section{Cells and reagents}

NCM460 and HCT116 cell lines were purchased from American Type Culture Collection. NCM460 cells are a type of normal colon cell, and HCT116 is a human colon cancer cell line. Both cell types were maintained in DMEM supplemented with $10 \%$ fetal bovine serum, $100 \mathrm{U} / \mathrm{mL}$ penicillin, and $100 \mu \mathrm{g} / \mathrm{mL}$ streptomycin. We used a polyclonal rabbit anti-DCLK1 antibody (ab37994; Abcam, Cambridge, UK) and a rabbit anti-ALDH1 mAb (ab134188; Abcam) as positive controls.

\section{Western blotting (WB) assay}

NCM460 and HCT116 cells were lysed in buffer containing $1 \%$ Triton X-100, 50 mM Tris- $\mathrm{HCl}$ (pH 7.5), $100 \mathrm{mM} \mathrm{NaCl}$, $50 \mathrm{mM}$ NaF, $5 \mathrm{mM}$ EDTA, $40 \mathrm{mM} \beta$-glycerophosphate, $1 \mathrm{mM}$ sodium orthovanadate, and $1 \mathrm{mM}$ phenylmethylsulfonyl fluoride for 30 minutes at $4^{\circ} \mathrm{C}$. CRC tissues were collected from patients after surgery and homogenized. Next, the tissues were lysed in lysis buffer in an ultrasonic machine. The samples were separated using 10\% sodium dodecyl sulfate-polyacrylamide gel electrophoresis and transferred to polyvinylidene difluoride membranes (T50093; Pall Corporation, New York, NY, USA). Subsequently, the membranes were blocked with 5\% nonfat milk and probed with the indicated primary antibodies $(2 \mu \mathrm{g} / \mathrm{mL})$ overnight. Then, they were washed three times with PBS containing $0.1 \%$ Tween 20 (PBST), incubated with a secondary antibody $(1: 5,000$, BL003A; Biosharp, Hefei, People's Republic of China) for 1 hour at room temperature, and then washed three times with PBST. The 47-kDa DCLK1-S protein was detected using electrochemiluminescence WB detection reagents (32109; Pierce, Rockford, IL, USA).

\section{Immunofluorescence assay}

HCT116 cells were plated on sterile glass cover slips. They were fixed with neutral 4\% paraformaldehyde, blocked, and permeabilized with 10\% normal goat serum (AR0009; Boster, Wuhan, People's Republic of China), 0.3\% bovine serum albumin (BSA), 0.05\% saponin, and 0.3\% Triton $\mathrm{X}-100$ in PBS for 1 hour. Next, the cells were incubated with primary antibodies ( $20 \mu \mathrm{g} / \mathrm{mL}$ for a final concentration) overnight at $4^{\circ} \mathrm{C}$ and stained with a fluorophore-conjugated secondary antibody (with the final concentration of $4 \mu \mathrm{g} / \mathrm{mL}$ for ab6717 and $2.5 \mu \mathrm{g} / \mathrm{mL}$ for ab7003; Abcam) for 1 hour at room temperature. After washing with ice-cold PBS, 2,4diamidino-2-phenylindole $(5 \mu \mathrm{g} / \mathrm{mL}$ for a final concentration, ZLI-9557; Zhongshan Golden Bridge Biotechnology, Beijing, People's Republic of China) in PBS was used to 
stain nuclei. A fluorescence microscope (Nikon Corporation, Tokyo, Japan) was used to capture images.

\section{Bacterial display analysis}

First, we cloned cDNA encoding the human DCLK1 sequence of 729-aa from a DCLK1 plasmid purchased from Kazusa (Tokyo, Japan). We then divided the sequence into seven regions that overlapped by 15 residues using specifically designed primers, which are listed in Table S1. The polymerase chain reaction (PCR) cycles were as follows: 3 minutes at $98^{\circ} \mathrm{C}$, followed by 15 cycles of 15 seconds at $98^{\circ} \mathrm{C}, 15$ seconds at $56^{\circ} \mathrm{C}$, and 1 minute at $72^{\circ} \mathrm{C}$. The seven partial PCR products were inserted into the pMD19-T expression vector and sequenced. Next, we changed the vector to pAPEx instead of pMD19-T and transformed the construct into DH $5 \alpha$ cells overnight. Bacteria were inoculated into $20 \mathrm{~mL}$ of fresh Luria-Bertani medium at $37^{\circ} \mathrm{C}$ with vigorous shaking (200 rpm) for $\sim 5$ hours until an optical density (OD) $578 \mathrm{~nm}$ of 0.7 was reached. We added $10 \mu \mathrm{L}$ of $0.1 \mathrm{mM}$ isopropyl- $\beta$-Dthiogalactopyranoside for another 4 hours of culture under the same conditions for protein synthesis. Then, 2-mL cultures were harvested based on the $\mathrm{OD}_{600 \mathrm{~nm}}$, washed three times with PBS, and centrifuged. The precipitate was used for further experiments. We then added $350 \mu \mathrm{L}$ of cold Tris-sucrose ( $0.1 \mathrm{~mol} / \mathrm{L}$ Tris- $\mathrm{HCl}$ [pH 8.0] and $0.75 \mathrm{~mol} / \mathrm{L}$ sucrose) solution to resuspend the bacteria, $35 \mu \mathrm{L}$ of fresh lysozyme $(10 \mathrm{mg} / \mathrm{mL})$ on ice, and $700 \mu \mathrm{L}$ of ice-cold EDTA $(1 \mathrm{mM})$ in a dropwise manner on a rotary shaker and placed the tube on ice for 15 minutes. Then, we added $50 \mu \mathrm{L} \mathrm{MgCl}_{2}(0.5 \mathrm{M})$ to the mixture on ice for another 20 minutes to obtain protoplasts, which expressed the protein of interest. After washing three times with PBS, the cells were incubated with anti-DCLK1 Abs $(5 \mu \mathrm{g} / \mathrm{mL})$ at $4^{\circ} \mathrm{C}$ for 1 hour, washed with PBS, and incubated with fluorescein isothiocyanate (FITC)-conjugated anti-rabbit $\operatorname{IgG}\left(1: 500\right.$, ab6717, Abcam) for 1 hour at $4^{\circ} \mathrm{C}$. After washing with PBS, the cells were analyzed with an FACSCanto ${ }^{\mathrm{TM}}$ flow cytometer (BD Biosciences, Franklin Lake, NJ, USA).

\section{Immunohistochemistry (IHC) assay}

Slides containing human CRC tissue sections were obtained from the Department of Pathology of Harbin Medical University. IHC analysis of CRC tissue sections on slides was performed using $20 \mu \mathrm{g} / \mathrm{mL}$ anti-DCLK1 (DCLK1-87) and anti-ALDH1 mAbs (1:250, ab134188; Abcam). A rabbit IgG antibody was used as a negative control (1:250, A7016; Biyuntian, Nanjing, People's Republic of China). Immunostaining was performed on $5-\mu \mathrm{m}$ slices of formalin-fixed paraffin-embedded tumor xenografts. Antigen retrieval was performed by microwave heating, and nonspecific proteinbinding sites were blocked with 4\% normal goat serum and $1 \%$ BSA in PBS for 1 hour. The 5- $\mu \mathrm{m}$ sections were incubated with anti-DCLK1 (DCLK1-87) and anti-ALDH1 mAbs (ab134188; Abcam). In parallel, nonimmune rabbit IgG was used as a negative control. A biotinylated secondary antibody, an avidin-biotinylated enzyme complex, and a 3,3'-diaminobenzidine substrate were used for detection (ZLI-9018; Zhongshan Golden Bridge Biotechnology). The slides were counterstained with hematoxylin and mounted with mounting medium for analysis. Images were captured using a Leica DM4000B microscope (Leica Microsystems, Wetzlar, Germany).

\section{Tissue microarrays and IHC scoring}

A tissue microarray (HCol-Ade180Sur-09) containing 100 paired samples of primary CRC tissues and adjacent tissues was obtained from Shanghai Outdo Biotech (Shanghai, People's Republic of China). The microarray was analyzed via IHC using the mAb DCLK1-87 $(20 \mu \mathrm{g} / \mathrm{mL})$. The correlation between DCLK1 expression and the clinical-pathological characteristics of CRC was statistically analyzed. Each stained microarray tissue section was scored independently and classified as D0-D3 according to the staining area and intensity. The DCLK $1^{+}$area was determined to be 0 for $0 \%-25 \%, 1$ for $25 \%-50 \%, 2$ for $50 \%-75 \%$, and 3 for $75 \%-100 \%$. The DCLK1 staining intensity was divided into grades $1,2,3$, and 4 from light to deep. We classified the DCLK1 expression level as 0-3 points (D0), 4-6 points (D1), 7-9 points (D2), or 10-12 points (D3) by multiplying the area and the immunostaining intensity. D0 and D1 were regarded as low DCLK1 expression, and D2 and D3 were regarded as high DCLK1 expression.

\section{Statistical analyses}

All statistical analyses were performed using GraphPad Prism Version 5.0 (GraphPad Software, Inc., La Jolla, CA, USA) or SPSS Statistics Version 19 (IBM Corporation, Armonk, NY, USA). For pairwise comparisons, the chi-squared test was used. For survival analysis, the log-rank test was used. A $P$-value $<0.05$ was considered statistically significant for all analyses.

\section{Results DCLKI -42 mAb and DCLKI-87 mAb bound specifically to DCLKI}

We generated two DCLK1-specific mAbs, DCLK1-87 mAb and DCLK1-42 mAb, using a novel chip-based immunospot 
array assay system. To determine the specificity of DCLK1-42 mAb and DCLK1-87 mAb, we performed WB assays. Previous studies have shown that DCLK1 appears as the DCLK1-L isoform at $82 \mathrm{kD}$ in normal colon cells and as the DCLK1-S isoform at $47 \mathrm{kD}$ in colon cancer cells, prompting us to use both cell lines to confirm the specificity of our antibodies. The results showed a band corresponding to DCLK1-L in NCM460 cells and a band corresponding to DCLK1-S in HCT116 cells. CRC tissues collected from CRC patients also showed a band corresponding to DCLK1-S (Figure 1A). We used DCLK1-42 mAb or DCLK1-87 mAb as the primary antibody, the polyclonal anti-DCLK1 antibody as a positive control, and the rabbit isotype IgG as a negative control.

To further characterize the cytosolic distribution of DCLK1 in HCT116 cells, we performed fluorescence immunocytochemistry assays. HCT116 cells were cultured on glass coverslips, immunostained with DCLK1-42 mAb or DCLK1-87 $\mathrm{mAb}$, and incubated with an FITC-conjugated antibody against rabbit IgG. We observed high levels of DCLK1 expression in HCT116 cells after DCLK1-42 mAb or DCLK1-87 mAb labeling. To further clarify the identity of DCLK1-positive cells, we performed double-labeling for DCLK1 and microtubules, as DCLK1 has been reported to interact with microtubules. The cells were visualized by fluorescence microscopy. We observed a high degree of colocalization between DCLK1 and microtubules in tumor cells (Figure 1B).

\section{Identification of the epitopes recognized by the DCLKI-42 and DCLKI-87} antibodies

The bacterial display method was used to identify the antigenic epitopes of DCLK1-42 mAb and DCLK1-87 mAb. DCLK1 is a transmembrane protein with a complex three-dimensional structure and multiple isoforms in various cell types. The use of larger DCLK1 fragments fo)r bacterial display retains the natural structure of DCLK1 as much as possible, which is important for identifying antibodies that bind to the native DCLK1 structure. We cloned full-length DCLK1 into seven pieces (Figure 2A), constructed prokaryotic expression vectors, transduced these vectors into bacteria, and performed flow cytometry analysis to identify specific binding.

We found that DCLK1-42 mAb binds to the first, second, and sixth regions, which corresponded to the 1-182-aa and 455-637-aa regions and that DCLK1-87 mAb binds to the seventh fragment, which is located in the 546-729-aa region of DCLK1 (Figure 2B, Table S2). These results showed that we obtained multiple epitope-specific rabbit mAbs against DCLK1, which should have potential clinical applications.

\section{Histological analysis of DCLKI+ cells in CRC tissues}

According to the epitope analysis, the DCLK1-87 mAb epitope was consistent with a commercial DCLK1 polyclonal antibody; thus, we used DCLK1-87 to perform IHC on CRC tissue slides and found that DCLK1-87 was more specific for DCLK1 expression in cells compared with a commercial anti-DCLK1 polyclonal antibody, which showed nonspecific binding in tissue stroma (Figure 3A). To evaluate the value of DCLK1-87 mAb in clinical applications, we performed IHC to confirm the potential of DCLK1 as a TSC marker and compared the immunostaining intensity with other valuable indexes used for prognostic CRC evaluation. ALDH1, which is a recognized colon TSC marker, was selected as a positive control. We examined histological sections of tumor tissues from patients exhibiting development of colorectal tubular adenocarcinoma. DCLK1-87 mAb and ALDH1 antibody were expressed at the bottom of intestinal glands. DCLK1 expression was relatively abundant and colocalized with ALDH1 expression, but DCLK $1^{+}$cells were more abundant and observed in a larger area range compared with $\mathrm{ALDH}^{+}$ cells (Figure 3B).

\section{DCLKI-87 mAb is a potential tool for the diagnostic and prognostic evaluation of CRC}

We performed IHC on tissue chips containing 100 paired samples of CRC and adjacent tissues from patients with 100-month follow-up data. Based on the DCLK1 immunostaining intensity, we classified the DCLK1 expression level as (-) (D0), (+) (D1), (++) (D2), or (+++) (D3) (Figure 4A). D0 and D1 were included in the DCLK1-low-expression group, whereas D2 and D3 were included in the DCLK1high-expression group. We found that $36.7 \%$ patients with CRC had high DCLK1 expression, although DCLK1 expression had no significant correlation with gender, age, pathological grade, clinical stage, TNM stages, tumor size, or tumor type in CRC patients (Table S3). However, patients with high DCLK1 expression exhibited longer survival time than patients with low DCLK1 expression $(P=0.0029$; Figure 4B), indicating that DCLK1 might play a protective role in $\mathrm{CRC}$ patients.

\section{Discussion}

DCLK1 has been reported to show high expression in many cancer cells and has been used as a cancer stem cell marker during murine tumorigenesis. ${ }^{7,14,16}$ A recent study found that a subset of DCLK $1^{+}$cells could renew cancer cells and have 
A

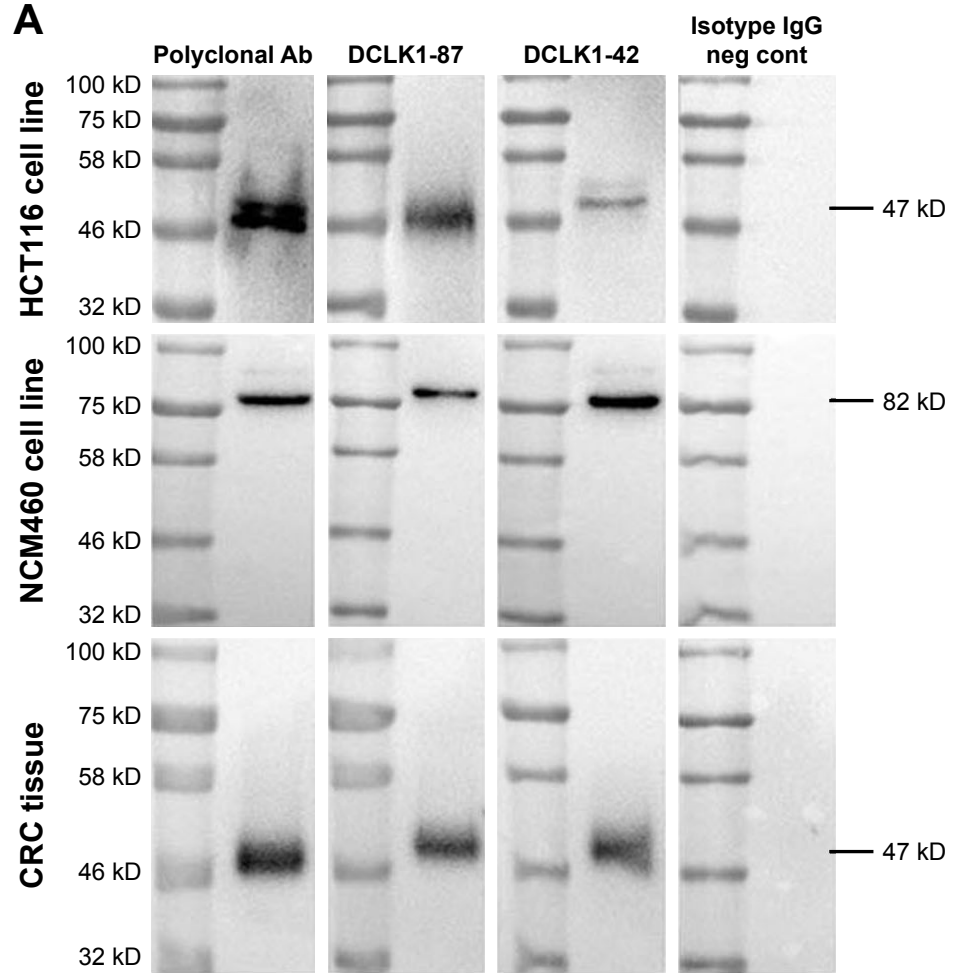

B

$40 \times$

DAPI
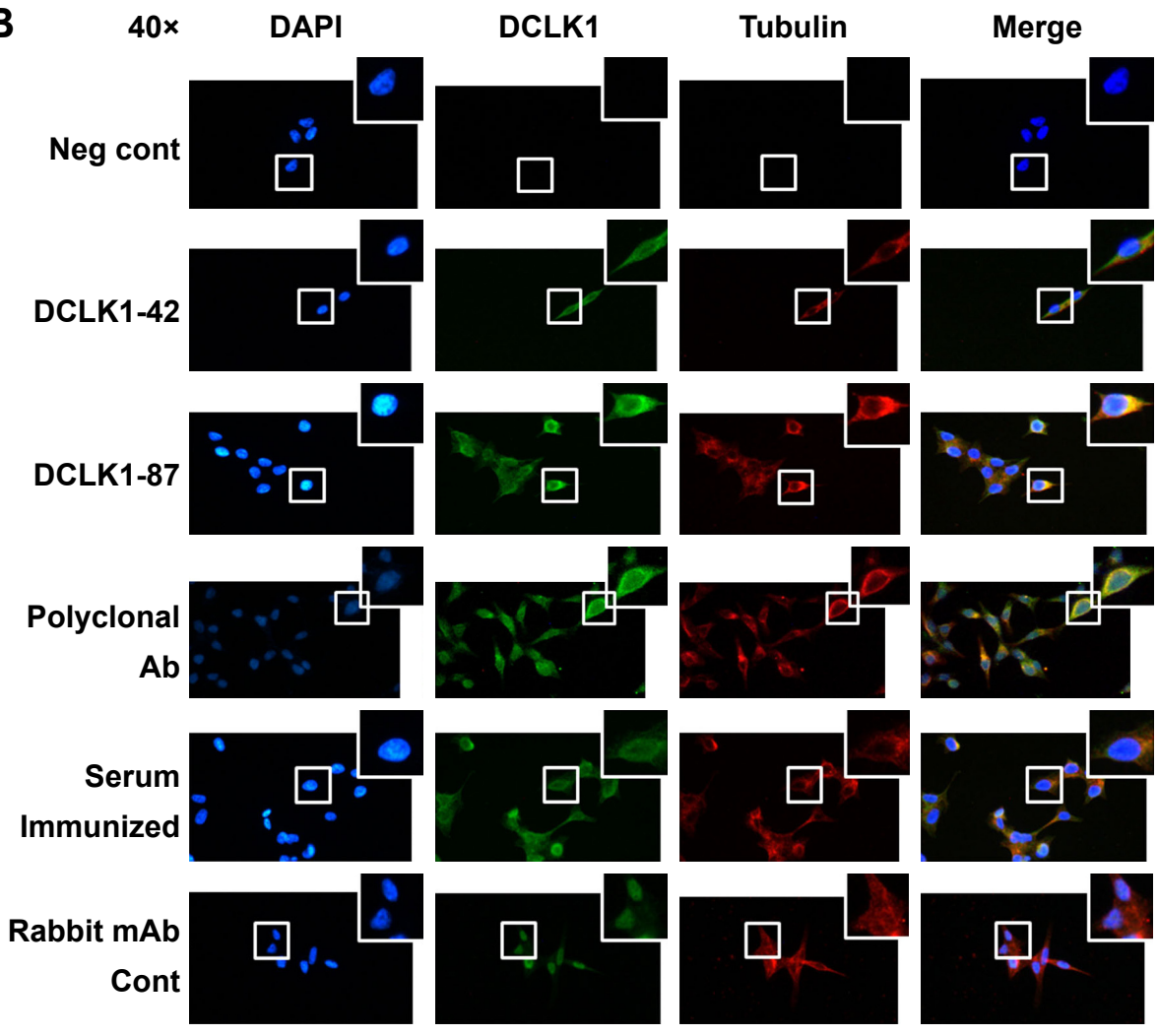

Figure I DCLKI-42 mAb and DCLKI-87 mAb specifically bound to DCLKI.

Notes: (A) DCLKI-42 mAb and DCLKI-87 mAb showed a $47 \mathrm{kD}$ band for DCLKI-S in HCTII6, and DCLKI-L in NCM460 was 82 kD. DCLKI from CRC tissues was detected by DCLKI-42 and DCLKI-87 at the size of DCLKI-S. (B) Localization analysis of DCLKI-42 mAb and DCLKI-87 mAb binding to DCLKI in HCTII6 cells. Immunofluorescence staining demonstrated that DCLKI-42 mAb and DCLKI-87 mAb (green) bound to DCLKI in the intracellular space and overlapped with microtubules (red). Negative control, no primary antibody; rabbit monoclonal negative control, an antinuclear rabbit monoclonal antibody used as a primary antibody; DCLKI-Abs, positive control, rabbit anti-DCLKI polyclonal antibody; serum, serum from a rabbit that received a fourth immunization with DCLKI.

Abbreviations: CRC, colorectal cancer; DCLKI, doublecortin-like kinase I; DCLKI-L, DCLKI-long; DCLKI-S, DCLKI-short; DCLKI-Abs, DCLKI antibodies; $\mathrm{mAb}$, monoclonal antibody; neg. cont, negative control; polyclonal Ab, polyclonal antibody; rabbit mAb Cont, rabbit monoclonal antibody control. 
A

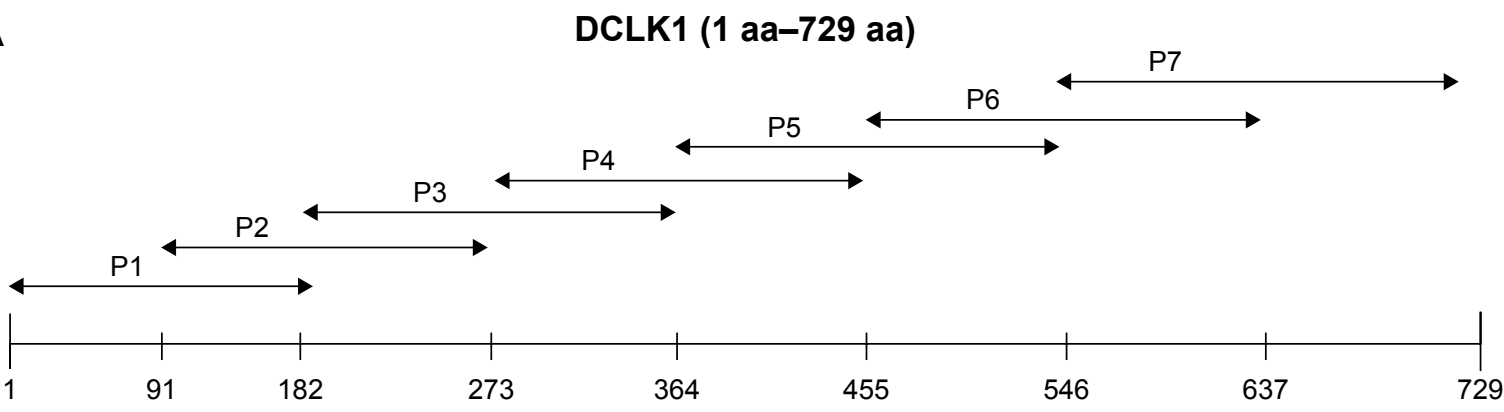

B

Serum
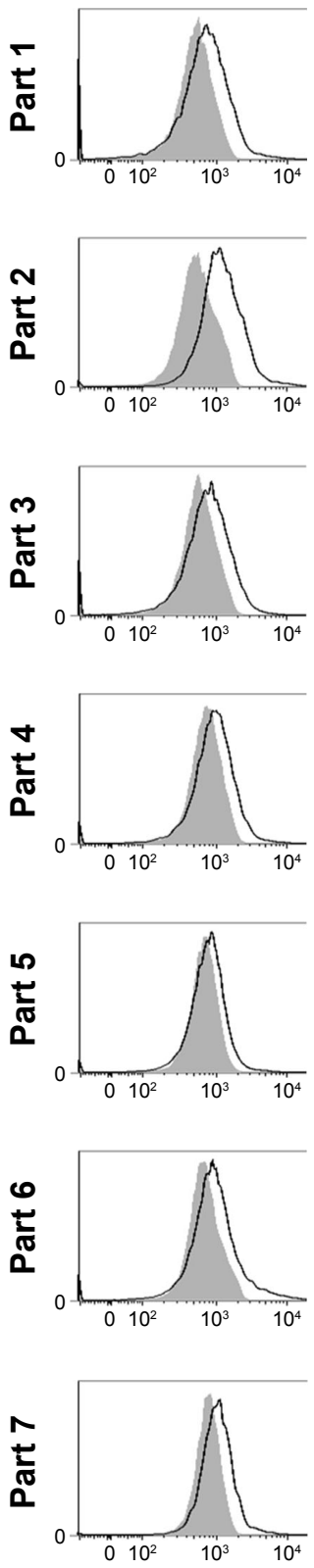

DCLK1-42
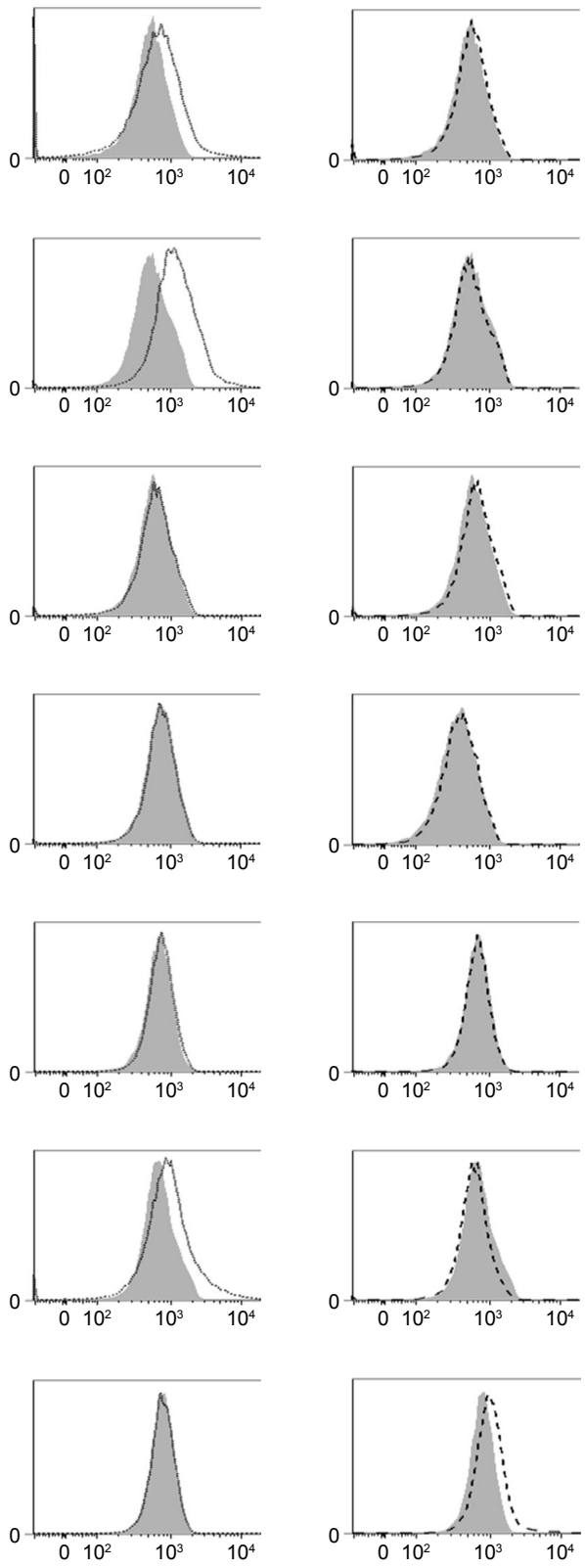

\section{DCLK1-87}
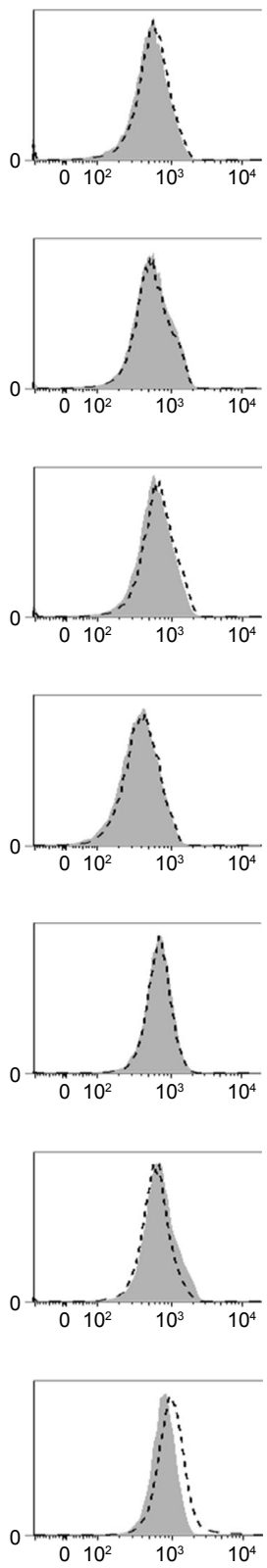

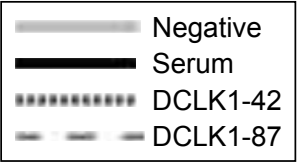

Figure 2 Preliminary determination of epitopes recognized by DCLKI-42 mAb and DCLKI-87 mAb.

Notes: (A) The DCLKI cDNA was cloned into a prokaryotic expression vector with seven pieces and then transduced into bacteria. (B) The antigen epitopes of DCLKI-42 $\mathrm{mAb}$ and DCLKI-87 mAb were determined via flow cytometry. Serum, rabbit serum from a rabbit that received a fourth immunization with DCLKI; negative control, no primary antibody.

Abbreviations: aa, amino acid; DCLKI, doublecortin-like kinase I; mAb, monoclonal antibody. 
A
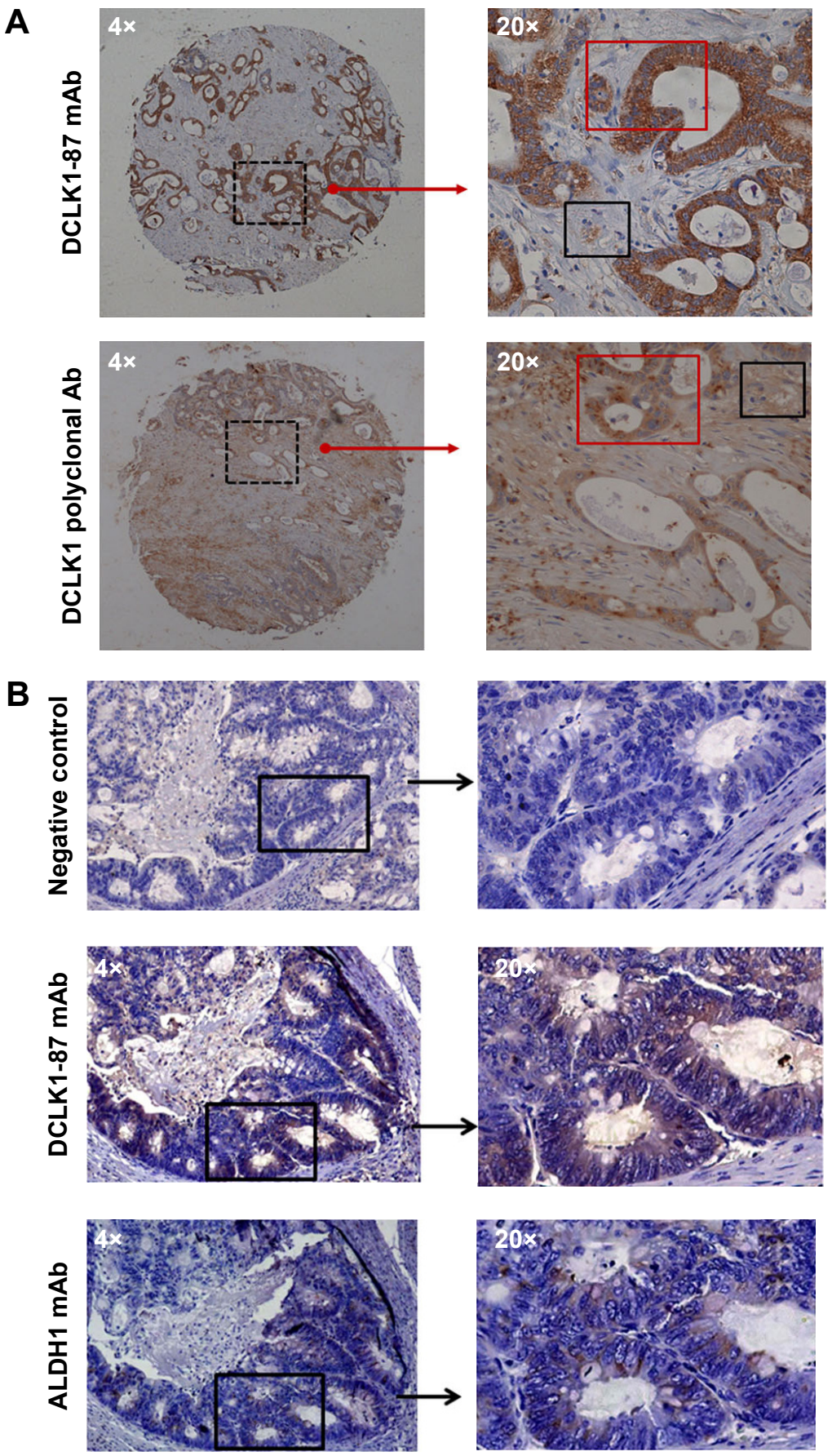

Figure 3 Histological analysis of DCLKI+ cells in CRC tissues.

Notes: (A) Comparison of the DCLKI-87 rabbit anti-DCLKI mAb and the rabbit polyclonal antibody in immunohistochemistry detection. Tumor biopsies from the same patient with CRC were stained with DCLK-87 mAb or the anti-DCLKI rabbit polyclonal antibody. The red box indicates the tumor cell area, and the black box with a solid line indicates the tissue stroma area. (B) Rabbit lgG was used as an isotype control. DCLKI-87 mAb was used as the primary antibody to detect DCLKI-expressing cells under low (4x; black box) and high (20x; right box) magnifications. A rabbit anti-ALDHI mAb was used as the primary antibody to detect ALDHI-expressing stem cells at low (4x; black box) and high (20x; right box) magnifications.

Abbreviations: Ab, antibody; CRC, colorectal cancer; DCLKI, doublecortin-like kinase I; mAb, monoclonal antibody.

the ability to resist chemotherapy. ${ }^{14}$ Thus, a tool for DCLK $1{ }^{+}$ cell monitoring is urgently needed. At present, many commercial anti-DCLK1 antibodies are polyclonal clones against the C-terminal end of DCLK1. Due to the disadvantages of these antibodies, such as nonspecific binding and instability between different batches of product, there should be better options for mAbs against DCLK $1 .{ }^{17}$ In addition, mAbs have potential as therapeutic agents. 
A

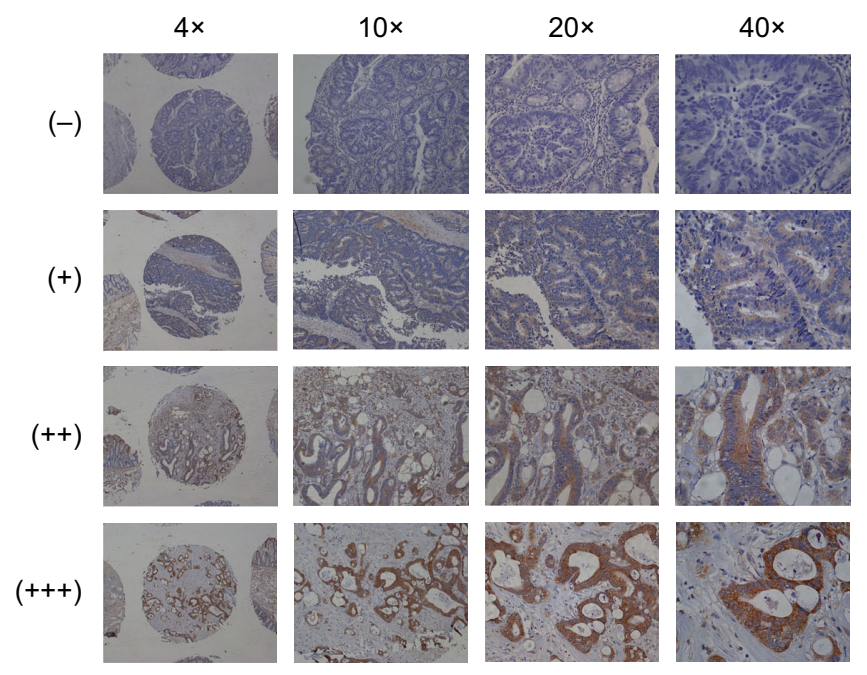

B

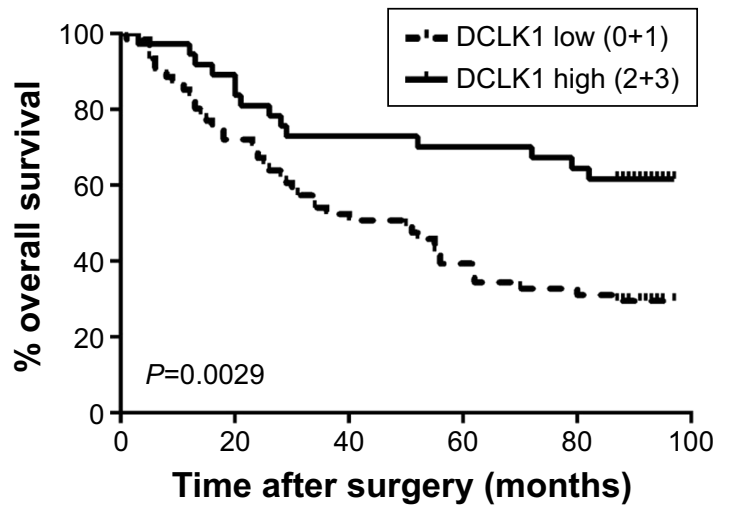

Figure 4 Overall survival and disease-free survival of CRC patients relative to low or high DCLKI expression.

Notes: (A) DCLKI expression was detected with DCLKI-87 mAb on a tissue microarray chip containing 100 paired samples of CRC tissues. The DCLKI expression intensity was classified into low- and high-expression groups: D0 (-) and DI (+) indicated low DCLKI expression; D2 (++) and D3 (+++) indicated high DCLKI expression. (B) Kaplan-Meier overall survival curves of CRC patients in relation to the relative expression intensity of DCLKI measured with DCLKI-87 mAb. $\mathrm{n}=100$ patients. Abbreviations: Ab, antibody; CRC, colorectal cancer; DCLKI, doublecortin-like kinase I; mAb, monoclonal antibody.

In this study, we used two pairs of DCLK1 mAbs generated previously for functional analysis. Previous reports showed that the majority of antibodies against DCLK1related epitopes are located in the regions near C-terminal end of DCLK1. Our results demonstrated that we obtained multiple epitope-specific mAbs against different regions of DCLK1. In particular, DCLK1-42 mAb exhibited features of a polyclonal antibody and bound to different epitopes. The results of WB confirmed the specificity of DCLK1-42 $\mathrm{mAb}$ and DCLK1-87 mAb in HCT116, NCM460, and CRC tissues. A high degree of overlap was observed between DCLK1 and microtubule protein expression. These results indicated that both DCLK1-42 mAb and DCLK1-87 mAb recognized DCLK1 in the cytoplasm and that DCLK1 was colocalized with microtubules. ${ }^{11}$

In an IHC test of CRC tissues, DCLK1-87 mAb showed more specific binding to DCLK1 $1^{+}$cells than a commercial polyclonal antibody, indicating that DCLK1-87 $\mathrm{mAb}$ could be used to reliably detect DCLK $1^{+}$cells. Furthermore, we opted to test the clinical applicability of DCLK1-87 mAb for DCLK1+ cancer cell monitoring and its potential for diagnostic and prognostic evaluations of CRC patients. IHC assays were performed on serial sections. DCLK1-87 mAb was used to detect DCLK1 ${ }^{+}$cells, and the confirmed colon TSC marker ALDH1 was used as a positive control. ${ }^{18}$ DCLK1 and ALDH1 were expressed in similar areas, but DCLK $1^{+}$cells were more abundant and located in a wider range than $\mathrm{ALDH} 1^{+}$cells. These results indicated that DCLK1 could be used as a tool for labeling TSCs and their progeny, which is consistent with other reports. $^{7}$

DCLK1 showed complex epigenetic modification in the promoter region of human CRCs, and previous studies have reported inconsistent results regarding the DCLK1 mRNA expression levels in human CRCs. ${ }^{14,19,20}$ In the database from Human Protein Atlas Project (www.proteinatlas.org), DCLK1 plays contrary prognostic marker roles in different types of human renal cancer, although another report showed that DCLK1 has a good prognostic impact on one type of human breast cancer. ${ }^{21}$ These studies suggested different functional roles for DCLK1 in different types of cancers. DCLK1 was reported to be highly expressed in gastrointestinal tumors and valuable as a marker for disease diagnostic and prognostic evaluation. ${ }^{19,22-24}$ We detected high DCLK1 expression in $36.7 \%$ of colorectal tissue samples based on a chip-based IHC assay using DCLK1-87 mAb as the primary antibody. Patients with high DCLK1 expression exhibited a significantly longer survival time than patients with low DCLK1 expression, indicating that DCLK1 might play a protective role in tumor progression. Studies have also indicated that DCLK $1^{+}$cells showed stem cell characteristics during tissue formation. ${ }^{16}$ In addition, DCLK1 has been revealed to play important roles in tissue repair, and DCLK1-knockout mice fail to maintain tight junctions. ${ }^{16,25,26}$ These functions of DCLK1 might support a protective role for DCLK1 in tumor progression. 


\section{Conclusion}

DCLK1-42 mAb and DCLK1-87 mAb generated by us were specific to DCLK1. They bound to not only DCLK1-L in normal colon cancer cells but also DCLK1-S in CRC cells and tissues. In particular, DCLK1-87 mAb was efficient for the precise detection of $\mathrm{DCLK} 1^{+}$cells in cancer tissues and has potential in the prognosis of CRC.

\section{Acknowledgments}

We thank the Translational Medicine Research and Cooperation Center of Northern China, Heilongjiang Academy of Medical Sciences of Harbin, People's Republic of China and the Key Laboratory of Infectious and Immunology of Heilongjiang Province for providing experimental environment. We also thank Prof Xiaoming Jin, Dr Yan He, and Dr Qi Huang from Department of Pathology, Harbin Medical University, for their excellent work and help in pathological analysis. This research was funded by Harbin Medical University, People's Republic of China.

\section{Disclosure}

The authors report no conflicts of interest in this work.

\section{References}

1. Burgess HA, Martinez S, Reiner O. KIAA0369, doublecortin-like kinase, is expressed during brain development. J Neurosci Res. 1999;58(4): $567-575$.

2. Koizumi H, Tanaka T, Gleeson JG. Doublecortin-like kinase functions with doublecortin to mediate fiber tract decussation and neuronal migration. Neuron. 2006;49(1):55-66.

3. May R, Riehl TE, Hunt C, Sureban SM, Anant S, Courtney WH. Identification of a novel putative gastrointestinal stem cell and adenoma stem cell marker, doublecortin and CaM kinase-like-1, following radiation injury and in adenomatous polyposis coli/multiple intestinal neoplasia mice. Stem Cells. 2008;26(3):630-637.

4. Sureban SM, May R, Lightfoot SA, et al. DCAMKL-1 regulates epithelial-mesenchymal transition in human pancreatic cells through a miR-200a-dependent mechanism. Cancer Res. 2011;71(6):2328-2338.

5. Ali N, Allam H, Bader T, et al. Fluvastatin interferes with hepatitis C virus replication via microtubule bundling and a doublecortin-like kinasemediated mechanism. PLoS One. 2013;8(11):e80304.

6. Vega KJ, May R, Sureban SM, et al. Identification of the putative intestinal stem cell marker doublecortin and CaM kinase-like-1 in Barrett's esophagus and esophageal adenocarcinoma. J Gastroenterol Hepatol. 2012;27(4):773-780.

7. Nakanishi Y, Seno H, Fukuoka A, et al. Dclk1 distinguishes between tumor and normal stem cells in the intestine. Nat Genet. 2013;45(1): 98-143.

8. Chandrakesan P, May R, Qu D, et al. Dclk1+ small intestinal epithelial tuft cells display the hallmarks of quiescence and self-renewal. Oncotarget. 2015;6(31):30876-30886.
9. Gerbe F, van Es JH, Makrini L, et al. Distinct ATOH1 and Neurog3 requirements define tuft cells as a new secretory cell type in the intestinal epithelium. J Cell Biol. 2011;192(5):767-780.

10. May R, Riehl TE, Hunt C, Sureban SM, Anant S, Houchen CW. Identification of a novel putative gastrointestinal stem cell and adenoma stem cell marker, doublecortin and CaM kinase-like-1, following radiation injury and in adenomatous polyposis coli/multiple intestinal neoplasia mice. Stem Cells. 2008;26(3):630-637.

11. Vreugdenhil E, Engels B, Middelburg R, et al. Multiple transcripts generated by the DCAMKL gene are expressed in the rat hippocampus. Brain Res Mol Brain Res. 2001;94(1-2):67-74.

12. Engels BM, Schouten TG, van Dullemen J, Gosens I, Vreugdenhil E. Functional differences between two DCLK splice variants. Mol Brain Res. 2004;120(2):103-114.

13. Sarkar S, Popov VL, O’Connell MR, et al. A novel antibody against cancer stem cell biomarker, DCLK1-S, is potentially useful for assessing colon cancer risk after screening colonoscopy. Lab Invest. 2017; 97(10):1245-1261.

14. O'Connell MR, Sarkar S, Luthra GK, et al. Epigenetic changes and alternate promoter usage by human colon cancers for expressing DCLK1-isoforms: clinical implications. Sci Rep. 2015;5:14983.

15. Ozawa T, Piao X, Kobayashi E, et al. A novel rabbit immunospot array assay on a chip allows for the rapid generation of rabbit monoclonal antibodies with high affinity. PLoS One. 2012;7(12):e52383.

16. Sureban SM, May R, Qu D, et al. DCLK1 regulates pluripotency and angiogenic factors via microRNA-dependent mechanisms in pancreatic cancer. PLoS One. 2013;8(9):e73940.

17. Waldmann TA. Monoclonal antibodies in diagnosis and therapy. Science. 1991;252(5013):1657-1662.

18. Ginestier C, Hur MH, Charafe-Jauffret E, et al. ALDH1 is a marker of normal and malignant human mammary stem cells and a predictor of poor clinical outcome. Cell Stem Cell. 2007;1(5):555-567.

19. Vedeld HM, Skotheim RI, Lothe RA, Lind GE. The recently suggested intestinal cancer stem cell marker DCLK1 is an epigenetic biomarker for colorectal cancer. Epigenetics. 2014;9(3):346-350.

20. Sarkar S, O'Connell MR, Okugawa Y, et al. FOXD3 regulates CSC marker, DCLK1-S, and invasive potential: prognostic implications in colon cancer. Mol Cancer Res. 2017;15(12):1678-1691.

21. Liu YH, Tsang JY, Ni YB, et al. Doublecortin-like kinase 1 expression associates with breast cancer with neuroendocrine differentiation. Oncotarget. 2016;7(2):1464-1476.

22. Gagliardi G, Moroz K, Bellows CF. Immunolocalization of DCAMKL-1, a putative intestinal stem cell marker, in normal colonic tissue. Pathol Res Pract. 2012;208(8):475-479.

23. Weygant N, Qu D, May R, et al. DCLK1 is a broadly dysregulated target against epithelial-mesenchymal transition, focal adhesion, and stemness in clear cell renal carcinoma. Oncotarget. 2015;6(4):2193-2205.

24. Gagliardi G, Goswami M, Passera R, Bellows CF. DCLK1 immunoreactivity in colorectal neoplasia. Clin Exp Gastroenterol. 2012;5:35-42.

25. May R, Sureban SM, Lightfoot SA, et al. Identification of a novel putative pancreatic stem/progenitor cell marker DCAMKL-1 in normal mouse pancreas. Am J Physiol Gastrointest Liver Physiol. 2010;299(2): G303-G310.

26. May R, Qu D, Weygant N, et al. Brief report: Dclk1 deletion in tuft cells results in impaired epithelial repair after radiation injury. Stem Cells. 2014;32(3):822-827. 


\section{Supplementary materials}

Table SI Primers used for the amplification of DCLKI peptide fragments

\begin{tabular}{|c|c|}
\hline Primer name & Primer sequence $\left(5^{\prime}-3^{\prime}\right)$ \\
\hline $\mathrm{PI}_{\text {Forward }}$ & GGCCCAGCCGGCCATGTCCTTCGGCAGAGACAT \\
\hline $\mathrm{PI}_{\text {Backward }}$ & GGCCCCCGAGGCCTTAGAAATCCTTATTCTCTCGC \\
\hline $\mathrm{P} 2_{\text {Forward }}$ & GGCCCAGCCGGCCTTGACCCGAACTCTGTCGG \\
\hline $\mathrm{P} 2_{\text {Backward }}$ & GGCCCCCGAGGCCTTATTCATCTAGCAAGAAATCAT \\
\hline$P 3_{\text {Forward }}$ & GGCCCAGCCGGCCTTCATTCGGCCCAAGCT \\
\hline $\mathrm{P} 3_{\text {Backward }}$ & GGCCCCCGAGGCCTTACGAGCTGCAGACTTTGG \\
\hline P4 ${ }_{\text {Forward }}$ & GGCCCAGCCGGCCGAAAGTGAATGTCGAGT \\
\hline P4 ${ }_{\text {Backward }}$ & GGCCCCCGAGGCCTTACATCTCCTCAATCAGAAG \\
\hline $\mathrm{P} 5_{\text {Forward }}$ & GGCCCAGCCGGCCTCGATGGATGAGAACGATGGCC \\
\hline $\mathrm{P} 5_{\text {Backward }}$ & GGCCCCCGAGGCCTTATGTGTACAGGGGGCCGTCTACAATG \\
\hline P6 Forward & GGCCCAGCCGGCCATGGATGTGCCAACTGAACT \\
\hline $\mathrm{P} 6_{\text {Backward }}$ & GGCCCCCGAGGCCTTAAGAAAATCGCTGATCTACAT \\
\hline P7 $7_{\text {Forward }}$ & GGCCCAGCCGGCCACAGTCTGTGGCACCCC \\
\hline P7 $7_{\text {Backward }}$ & GGCCCCCGAGGCCTCACATCCTGGTTGCGTCT \\
\hline
\end{tabular}

Abbreviation: DCLKI, doublecortin-like kinase I.

Table S2 Epitopes of the DCLKI-42 and DCLKI-87 antibodies

\begin{tabular}{llllllll}
\hline DCLKI & Part & Part 2 & Part 3 & Part 4 & Part 5 & Part 6 & Part 7 \\
fragments (aa) & I-182 & 9I-273 & $182-364$ & $\mathbf{2 7 3 - 4 5 5}$ & $\mathbf{3 6 4 - 5 6 4}$ & $\mathbf{4 5 5 - 6 3 7}$ & $\mathbf{5 4 6 - 7 2 9}$ \\
\hline DCLKI-42 & 0 & $\bigcirc$ & $\times$ & $\times$ & $\times$ & 0 & $\times$ \\
DCLKI-87 & $\times$ & $\times$ & $\times$ & $\times$ & $\times$ & $\times$ & 0 \\
\hline
\end{tabular}

Notes: $\bigcirc$, binding; $\times$, no binding.

Abbreviations: aa, amino acid; DCLKI, doublecortin-like kinase I.

Table S3 DCLKI expression and clinical characteristics of the patients with CRC

\begin{tabular}{|c|c|c|c|c|}
\hline \multirow[t]{2}{*}{ Characteristics } & \multirow[t]{2}{*}{$\mathbf{n}$} & \multicolumn{2}{|c|}{ DCLKI expression } & \multirow[t]{2}{*}{$P$-values } \\
\hline & & Low (\%) & High (\%) & \\
\hline Gender & & & & 0.7773 \\
\hline Female & 53 & 34 & 19 & \\
\hline Male & 44 & 27 & 17 & \\
\hline Age (years) & & & & 0.75915 \\
\hline$\leq 65$ & 35 & 22 & 13 & \\
\hline$>65$ & 57 & 34 & 23 & \\
\hline Pathological grade & & & & 0.78419 \\
\hline I-II & 57 & 36 & 21 & \\
\hline III-IV & 41 & 27 & 14 & \\
\hline Clinical stage & & & & 0.59867 \\
\hline$\leq 2 b$ & 57 & 35 & 22 & \\
\hline$>2 b$ & 39 & 26 & 13 & \\
\hline $\mathrm{T}$ & & & & 0.56566 \\
\hline TI-T3 & 82 & 57 & 25 & \\
\hline $\mathrm{T} 4$ & 13 & 8 & 5 & \\
\hline $\mathrm{N}$ & & & & 0.56157 \\
\hline $\mathrm{N} 0+\mathrm{NIa}$ & 70 & 44 & 26 & \\
\hline $\mathrm{NIb}+\mathrm{N} 2$ & 26 & 18 & 8 & \\
\hline M & & & & 0.92873 \\
\hline Mo & 95 & 61 & 34 & \\
\hline $\mathrm{MI}$ & 3 & 2 & I & \\
\hline Tumor size $(\mathrm{cm})$ & & & & 0.42809 \\
\hline$\leq 5$ & 49 & 34 & 15 & \\
\hline$>5$ & 47 & 29 & 18 & \\
\hline Tumor type & & & & 0.63227 \\
\hline Tubular adenocarcinoma & 46 & 31 & 15 & \\
\hline Mucous adenocarcinoma & 51 & 32 & 19 & \\
\hline
\end{tabular}

Note: The $P$-values were obtained from the chi-squared test.

Abbreviations: CRC, colorectal cancer; DCLKI, doublecortin-like kinase I. 
OncoTargets and Therapy

\section{Publish your work in this journal}

OncoTargets and Therapy is an international, peer-reviewed, open access journal focusing on the pathological basis of all cancers, potential targets for therapy and treatment protocols employed to improve the management of cancer patients. The journal also focuses on the impact of management programs and new therapeutic agents and protocols on The manuscript management system is completely online and includes a very quick and fair peer-review system, which is all easy to use. Visit http://www.dovepress.com/testimonials.php to read real quotes from published authors.

Submit your manuscript here: http://www.dovepress.com/oncotargets-and-therapy-journal 